\title{
THERA: Two-level Hierarchical Hybrid Road-Aware Routing for Vehicular Networks
}

\author{
Muhammad Tahir Abbas ${ }^{1}$ and Wang-Cheol SONG ${ }^{2 *}$ \\ Department of Computer Engineering, Jeju National University \\ Jeju, South Korea \\ 1e-mail: htahir6200@gmail.com] \\ [e-mail: kingiron@gmail.com] \\ ${ }^{2}$ Corresponding Author: Wang-Cheol SONG
}

Received October 30, 2017; revised March 9, 2018; revised June 11, 2018; revised Augsut 8, 2018; accepted January 17, 2019; published July 31, 2019

\begin{abstract}
There are various research challenges in vehicular ad hoc networks (VANETs) that need to be focused until an extensive deployment of it becomes conceivable. Design and development of a scalable routing algorithm for VANETs is one of the critical issue due to frequent path disruptions caused by the vehicle's mobility. This study aims to provide a novel road-aware routing protocol for vehicular networks named as Two-level hierarchical Hybrid Road-Aware (THERA) routing for vehicular ad hoc networks. The proposed protocol is designed explicitly for inter-vehicle communication. In THERA, roads are distributed into non-overlapping road segments to reduce the routing overhead. Unlike other protocols, discovery process does not flood the network with packet broadcasts. Instead, THERA uses the concept of Gateway Vehicles (GV) for the discovery process. In addition, a route between source and destination is flexible to changing topology, as THERA only requires road segment ID and destination ID for the communication. Furthermore, Road-Aware routing reduces the traffic congestion, bypasses the single point of failure, and facilitates the network management. Finally yet importantly, this paper also proposes a probabilistical model to estimate a path duration for each road segment using the highway mobility model. The flexibility of the proposed protocol is evaluated by performing extensive simulations in NS3. We have used SUMO simulator to generate real time vehicular traffic on the roads of Gangnam, South Korea. Comparative analysis of the results confirm that routing overhead for maintaining the network topology is smaller than few previously proposed routing algorithms.
\end{abstract}

Keywords: Road-Aware routing, VANETs, Geographic routing, Hybrid, Road segments, Intelligent transport system (ITS) 


\section{Introduction}

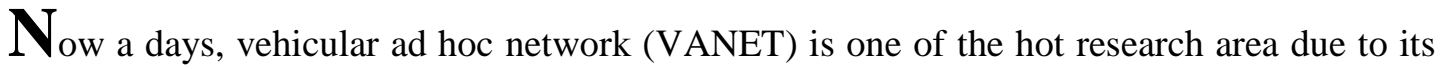
encouraging solutions to the Intelligent Transport System (ITS). VANET turns each participating vehicle into a mobile node or a wireless router, permitting vehicles to associate with each other and, in turn, create a network with a wide range. Connected vehicles can have a link breakdown periodically because of the short lifespan of communication links and uncertain driver's actions. Owing to these aspects of VANETs, it turns into a primary issue to offer an effective routing protocol that can accord with the dynamic behavior of VANETs.

As far as vehicular movement is concerned, vehicles are bound to roads only. So, a road-aware routing technique is required in order to reduce the bandwidth utilization by the usage of road statistics efficiently. A road between intersections/crossings can be considered as a segment or a zone. Vehicles available on the same segments mainly have identical communication conditions/characteristics. The change of vehicle topology can be managed efficiently on the local road segment without the usage of whole network bandwidth if the routing information is acknowledged separately.

In the literature, a number of routing protocols have been anticipated so far. Over the past few years, geographic routing protocols [1] [2] [3] [4] [5] [6] [7] are studied categorically to accord with maneuverability in VANETs. Geographic routing protocols are considered scalable with respect to the proportion of the network as these protocols are not required to manage routing tables at each node. This is only the reason that these protocols are contemplated as best candidate for inter-vehicle communication. However, most of them use the global view of the simulator and location of destination is already known. Therefore, these protocols have zero location service overhead. Furthermore, for delay sensitive applications, the performance of geographic routing protocols still requires to be improved in terms of end-to-end delay and packet delivery ratio.

All the protocols in VANETs follows an approach of packet forwarding, either towards the next hop node or an intersection. A simplest and easy way is through the greedy approach, however, it does not guarantee the packet delivery. In addition, after making a number of developments the problem of packet delivery still there but with little probability [3]. In [4], a novel traffic flow-based geographic routing protocol (TFOR) is proposed to enhance the road safety and the traffic flow by an intelligent transportation system. TFOR operates in two parts, firstly with selection of intersection and then information forwarding using the greedy forward approach.

In this paper, we present a novel Road-Aware hierarchical position-based routing protocol called Two-level Hierarchical Hybrid Road-Aware (THERA) protocol, designed especially for inter-vehicular communication for an urban and highway environment. Roads are divided into non overlapping road segments. Aggregating vehicles into road segments conceals the detail of the network topology. At the start, vehicles know their location and therefore their road ID through Global positioning System (GPS). THERA incorporates discovering destination with determining possible routes between source and destination. Vehicles near the intersection are responsible for maintaining a communication link between different road segments and are termed as Gateway vehicles (GV) or gateway nodes. Destination discovery does not consider the network wide packet broadcasts instead of that GV only forward discovery packets from one road segment to another. Inside the road segment, path with maximum duration is adopted for the communication. In addition, a mathematical analysis is performed by the derivation of probability density function $(p d f)$ for the calculation of reliable 
and durable path between two intersections. Once a complete route is available, it is accommodated on the fly to account for any changes in the topology. THERA is a hybrid protocol as it combines both the approaches, proactive and reactive, at a same time.

The key contribution of this paper is summarized as follows:

- The new taxonomy of geographic based road-aware routing for vehicular networks is provided in this paper.

- Partitioning: In order to reduce the routing overhead, roads are divided into road segments with unique ids.

- Gateway vehicles: Introduced a concept of gateway vehicles to provide the connectivity between the road segments and to forward the RREQ and data packets to the other segments in the meanwhile acting as a bridge.

- Proactive and Reactive approach: THERA adopts a hybrid approach for maintaining intra-road segment table and for route discovery mechanism.

- Packet forwarding: RREQ is only forwarded by the GV instead of the normal vehicles

- Path recovery: With the help of gateway vehicles, link to the destination can be reestablished again without starting new discovery process.

- Mathematical model: A probabilistic mathematical model is provided for the estimation of path duration inside each of the road segments.

- Parameters for mathematical model: The following parameters are used for the estimation of path duration:

o Hop count

o Transmission range

o Velocity

o Area for the next hop node

The rest of the paper is structured as follows. Section 2 presents the related work. A complete overview of THERA, its working is provided in section 3 and 4 respectively. In section 5, a probabilistic model is derived to estimate path duration between two road intersections. In the second half, paper greatly focuses on results which are presented in section 6 . Finally the conclusion is made in section 7.

\section{Related Work}

Numerous applications for VANETs have been proposed [19], which uses multi-hop connection, so a routing protocol is required to adopt fast changes of network topology. There are some Geographic protocols [7] [8] that provides the shortest route between the source and the destination. Nevertheless, it is not always feasible that the shortest route is populated between source and destination. Moreover, a new route is calculated if the local maximum is reached at any point. This process may waste network capacity and may take some extra time. Generally, before starting the data forwarding process GR protocols require the location information of the destination. Most of the GR based proposed protocols assume the destination position is known at any time [11] [12] [13]. These protocols are evaluated with zero location service overhead and shows good performance. On the other hand, it still remains unclear how the destination location discovery procedure can clout the network bandwidth. In addition, these protocols fail if the end point vehicle travels a significant distance from its acknowledged position. 
In position-based routing protocols, normally it is assumed that physically adjacent vehicles are also closed in the network topology. So a data packet is forwarded frequently, based on the same assumption, to a geographically closest neighbor until its delivery to the destination. However, the assumption characterized above does not hold true in VANETs where geographical distribution of vehicles is barred by the road infrastructure.

RTR (Road Topology-aware Routing) [8] expands the route discovery process of Ad hoc on demand distance vector routing (AODV) by the discovery of routing paths with two junctions-disjoints. For data dissemination, the source node selects a path instead of selecting all for one packet forwarding in order to reduce the traffic overhead. The path is adopted alternately for the communication if both two junction-disjoint paths are connected. Utilization of two junction-disjoint paths for data dissemination reduces the link failure and network congestion in a single routing path. In RTR, control overhead for destination discovery is even more than that of AODV. This is because in RTR, the RREP is sent back to the source from the destination over two different paths.

iCAR [9] (intersection-based Connectivity Aware Routing) protocol is a position-based protocol for VANETs. An optimized broadcast with the consideration of packet delivery delays at road segments and a real-time traffic, is used in it. For the selection of next segment, iCAR estimates the neighbor road segments for highest density, less distance towards the destination and minimum delay. iCARII [10] assume that every vehicle is equipped with the global positioning system. Proposed protocol enhance the protocol performance with the improvement of packet delivery ratio by taking into account the fixed infrastructure and one-hop broadcast of beacon packets in order to periodically update the driving conditions. iCARII is further divided into four segments which are next hop selection, selection of neighbor intersection, verity period calculation and segment assessment. In the first phase of segment assessment, protocol collects the real-time information of the entire network. In the second phase of verity period estimation, proposed protocol computes path life time for intersections and traversing segments after receiving a number of control packets. In third phase, existence of updated path towards the destination is determined by the selection of neighbor intersection. In the last phase, if a packet face sudden path failure, a new request is made by the current vehicle for a new path towards the centralized location servers.

The approach of "Gateway Nodes" is not completely new. Gateway node is used to maintain connectivity between zones. There can be more than one gateway nodes between multiple zones, therefore, it bypasses single point of failure. An identical approach is adopted in UGAD [14], where vehicles are given the highest priority in order to avoid the transmission blocking by the buildings near intersections. Near the intersections, vehicles can send/receive packets to/from other connected roads segments. We concede using these vehicles in an effective manner can enhance the performance of the routing protocol.

\section{Two-level hierarchical Road-Aware routing}

In this study, we present a new position-based Hierarchical hybrid protocol called Two-level Hierarchical Hybrid Road-Aware (THERA), designed conspicuously for vehicular networks. Two levels of network topology are defined in THERA: Intra-road segment level (First level of hierarchical model) topology and inter-road segment level (2nd level of hierarchical model) topology. In THERA a physical link exists if any two vehicles on the same road segment are within the transmission range (Fig. 1 (a)). In the first level of hierarchical model, vehicles generate link state packets (LSPs) containing their neighbor information to 
make a road segment routing table. This proactive mechanism is only used for the routing on road segments between intersections. The first level of hierarchical model provides the knowledge about the connected vehicles through available links.

In Fig. 1 (a), if a vehicle B wants to forward a data packet to E, the packet has to send through the vehicles A-F using intra-road segment routing table. However, inter-road segment topology shows road segment road segment connectivity. Various road segments are connected if there is at least one physical link exists Fig. 1 (b) between two roads.

In the second layer of THERA, enhanced reactive routing approach is used to identify a path to destination, as shown in Fig. 1(b). The path request is not forwarded to every single vehicle instead it is only sent to a limited vehicles on each road segment. After a path is completely discovered, it is readjusted on the fly to account for modifications in the topology without initiating a new destination discovery request. To minimize the control routing overhead, THERA does not flood the network with the packet broadcasts for destination discovery process. In Fig. 1(b), a communication link exists between road segments 1 and 3 are 1-4-3. In the next section, we will see how a vehicle adopt intra-road segment topology to forward a data packet within a road segment and how it adopts for inter-road segment topology to forward a data packet between road segments.

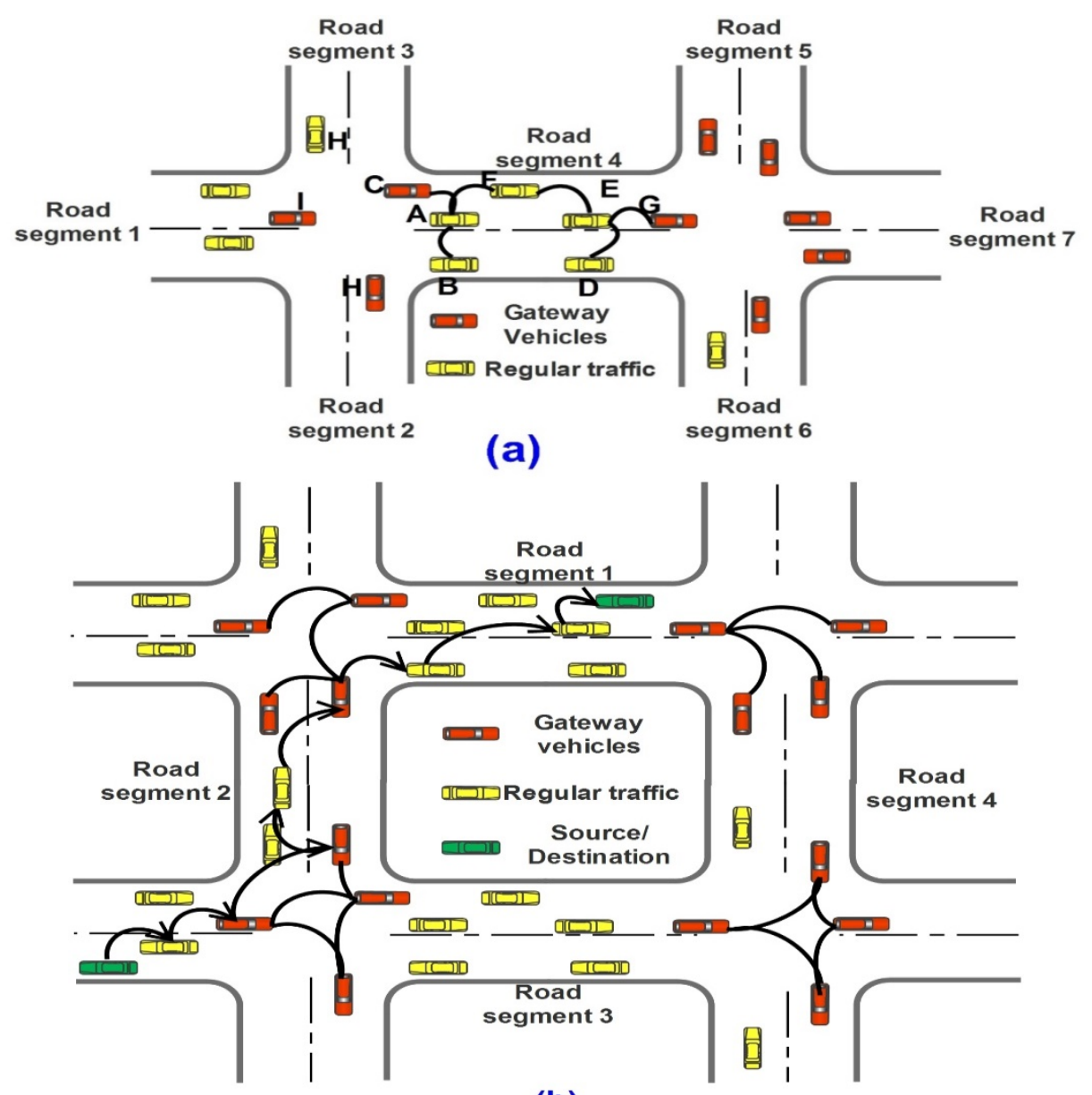

(b)

Fig. 1. Discovery Process (a) Intra-Road segment topology with gateway vehicles (b) Inter-Road segment RREQ 
In order to expedite THERA, every vehicle hears two kinds of link state packets proactively and reactively that include intra-road segment LSP's and inter-road segment RREQ/RREP packets, respectively. In the first level of hierarchical model, intra-road segments LSP's carry an information of connected vehicles inside the road segment and propagated locally within a same segments (Fig. 1(a)) in order to obtain an intra-road segment routing table. The procedure of maintaining routing tables proactively is only used for roads between crossing/intersections. However, a path between source and destination is discovered and maintained reactively at the second level of hierarchical model. During the route discovery, route request is not forwarded to every vehicle in the network except few vehicles near the crossings named as Gateway vehicles (GV).

Table 1. Intra-Road segment Neighbor table

\begin{tabular}{|c|c|}
\hline Source & Neighbors \\
\hline \hline B & A \\
\hline C & A, F, 1, 2, 3 \\
\hline D & E \\
\hline E & F, D, G \\
\hline F & E, C, A \\
\hline A & F, C, B \\
\hline G & E, 5, 6, 7 \\
\hline
\end{tabular}

Table 2. Intra-Road segment 1 routing table for Vehicle $\mathrm{E}$

\begin{tabular}{|c|c|}
\hline Destination & Next hop node \\
\hline A & F \\
\hline B & F \\
\hline C & F \\
\hline D & D \\
\hline F & F \\
\hline G & G \\
\hline 3 & F \\
\hline 4 & G \\
\hline 2 & F \\
\hline
\end{tabular}

As described in the previous section, In THERA, roads are divided into non-overlapping road segments with unique IDs. Vehicles, belong to the same road segment, initialize the process of making intra-road segment routing table.

\subsection{Intra-Road Segment Routing}

Every vehicle on the road dynamically broadcasts a link request to determine its neighbor vehicles. Neighbors inside the transmission range in turn reply with link packet that contains $<$ road id, vehicle id, position, direction, speed $>$. While forwarding a data packet towards the destination, a sender vehicle always includes a road id instead of vehicle id. Purpose of including a road id is that the vehicle may change its direction and movement during a data transmission, results in the disconnection of a link. Therefore, even after the link failure between any two intermediate vehicles, the data can still be forwarded by other vehicles using their road segment routing table. After hearing LSP's from neighbors, every vehicle then inseminate their neighbor LSP inside the same road segment by intermediate vehicles. For example, in Fig. 1(a), vehicles F, C and B are the neighbors of the vehicle A. As every vehicle 
performs same process, a table of intra-road Segment neighbor LSP's, such as the one shown in Table 1, can be stored in every vehicle.

Vehicles near the intersection are selected as a forwarding node are named as gateway vehicles (GV). In a city scenario, there can be multiple vehicles near the intersection, so selection of GV is performed accordingly. More than two vehicles can be selected as a GV at each road segment and it depends on the availability of vehicle. Every vehicle will know the intra-road Segment topology after receiving LSP's from all the vehicles. In order to create intra-road segment routing table, shortest path approach is carried out. Intra-road segment table for vehicle $\mathrm{E}$ is given in Table 2. Due to vehicular movement and channel fading, the process of LSP's propagation has to be executed repeatedly to observe any change in communication link. If a vehicle moves to another road segment, its intra-road segment routing table would be left in its old segment. For this, a timer 'TRS' is set for every received neighbor/vehicle LSP and the expired one will be deleted after waiting for 'TRS' time when the vehicle moves out of its range.

\subsection{Inter-Road Segment Routing}

Vehicles may receive link responses from the vehicles of their connected segments. These vehicles are termed as gateway nodes. Vehicles $C$ and G in Fig. 1(a) (red vehicles) are GV of Road segment 1 . In THERA, GV provides the connectivity between two neighbor road segments. In order to discover a route to destination, THERA uses the mechanism of route request/reply RREQ/RREP, as shown in Fig. 1(b). Source vehicle checks its intra-road segment routing table, before sending the RREQ, to find the destination vehicle if both are on the same road segment. Source vehicle sends a route request to $\mathrm{GV}$ if the destination vehicle is on a different road. The RREQ, on the intermediate roads, is forwarded from one GV to others using the road segment routing table. RREQ will only be forwarded by the Gateway vehicles to the other road segments. However, if a normal vehicle receives RREQ, it will be forwarded towards the GV once and will be dropped after receiving another packet with the same id. Gateway vehicles, upon the reception of RREQ, search their road segment routing tables to locate the destination vehicle. GV with the destination on the same road segment then reply with RREP to the source vehicle including the Road ID, which represent the direction of the traffic flow and hence the direction of the destination vehicle is also known.

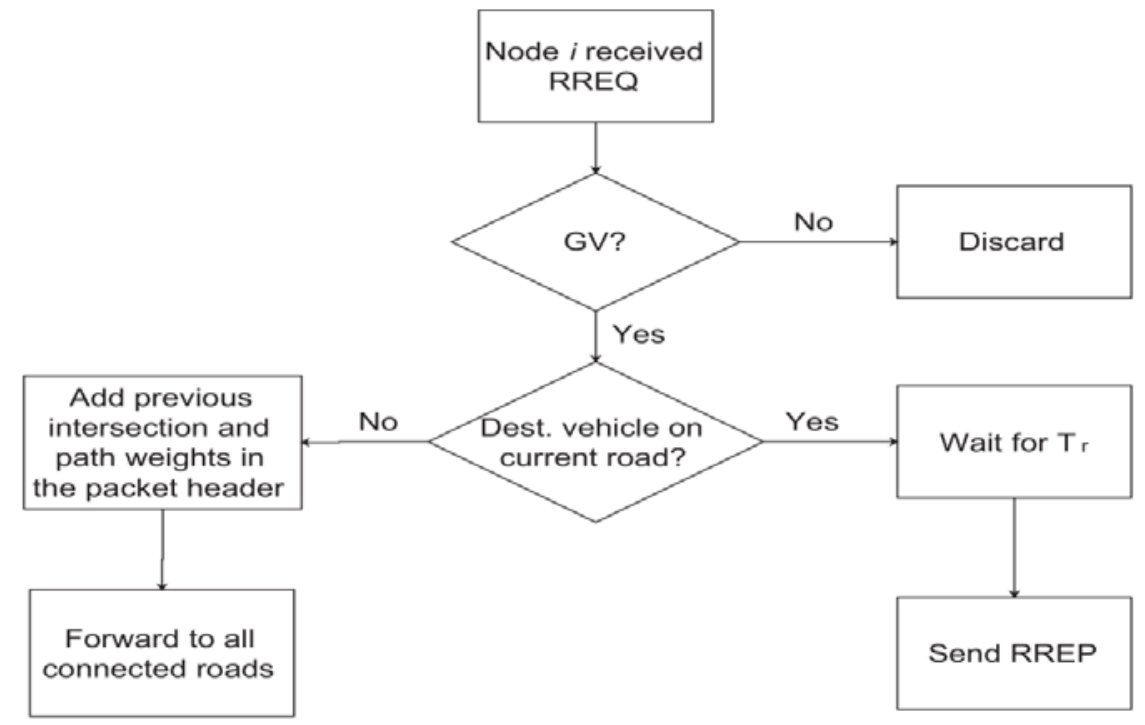

Fig. 2. RREQ processing flowchart 
Routing protocols with RREQ/RREP approach includes vehicle ID in the RREP. However, THERA adds a road ID rather than a vehicle ID in their RREP packets. The Reason behind adding the road ID in RREP instead of vehicle ID is that a path between two vehicles in the route may fail due to sharp turns resulting in decreased performance. Therefore, even after the link failure between any two intermediate vehicles, other vehicles can still forward the data packets using their intra-road segment routing table.

\subsection{Data transmission over discovered route}

The THERA, data packets are forwarded towards the destination using road segment routing table. Gateway vehicles are responsible for packet transmission from one intersection to another. As beaconing is an essential part for communication protocols in VANETs, THERA expand this approach to set up the intra-road segment routing tables. Using intra-road segment routing tables, intermediate vehicles route the packet to next gateway vehicle. An example packet forwarding by intermediate vehicle can be seen in Fig. 1(b).

\subsection{Path maintenance using Gateway Vehicles}

In THERA, during the data transmission, a table is maintained on both ends (Destination and Source) by the gateway vehicles. A communication link between the source and the destination remain valid only if both the end nodes do not change their corresponding road segments. If any of the end node travels to road segment, then the GV of the previous road segment finds it out from the road segment routing table as the table is updated periodically. Let's assume that a path within a road segment stays connected. Intersections and different road segments are thus only the possibility for a link breakage between a source and the destination.

If any of source/destination vehicle navigates a significant distance from its current location (or makes a sharp turn at an intersection), previously proposed protocols fail with the initialization of new discovery process. At this point we will explain that how the GV assists to accommodate to such a state without losing data packets and avert a new route discovery procedure. Before leaving a road segment, vehicle notifies GV nodes by broadcasting a road_segment_leave packet. Leaving node also needs to notify the GV with the road ID after moving into a new road segment by considering the old road segment GV as a destination. Right after receiving a first notification from leaving node, GV updated its intra-road segment table accordingly. Every time a GV vehicle receives data packet for a node that notifies the GV before, the gateway vehicle retransmits the packet after making an update to the packet header with the node's current road ID. Furthermore, GV also sends an updated route to the source vehicle.

In THERA, without the initializing a discovery process from start, a massive network bandwidth can be protected. Without employing a new path discovery, GVs help adjusting the connected path even if the destination change its direction and/or moving speed.

\subsection{Path duration estimation}

THERA integrates the features of reactive and proactive routing concurrently while guaranteeing the availability of a reliable path between two intersections. Every vehicle on the same road segment perceives link availability and node connectivity by the proactive approach. In our approach, link quality within a road segment is calculated by the estimation of path duration using different parameters that include hop count for the transmission, link 
availability time and vehicle velocity. This technique of local information sharing reduces the extra overhead while improving the process of link discovery over a wireless network. Furthermore, our proposed mathematical model for path estimation (Mathematical model is explained well in our previous paper [15]) guarantees link reliability and stability for a road segment.

Route with less number of hops is considered for path estimation, as it robustness against vehicle mobility. To find the neighbor node at the extreme end of transmission range, as shown in Fig. 3, the following equation is derived:

$$
\begin{gathered}
A_{\text {Total }}=A_{\text {Int } 1}+A_{\text {Int } 2} \\
A_{\text {Total }}=\left[\frac{(\alpha-\sin (\alpha)) \cdot R_{s}^{2}}{2}\right]+\left[\frac{(\beta-\sin (\beta)) \cdot R_{s}^{2}}{2}\right]
\end{gathered}
$$

Where $A_{\text {Int1 }}$ and $A_{\text {Int2 }}$ represents the area for finding a next hop node.

For the estimation of path duration in VANETs, velocity is one of the important design parameter. Let's assume $V_{S}$ and $V_{N H}$ be the velocity of source and next hop node respectively. Then the relative velocity can be calculated as:

$$
\left|R_{V}\right|=\sqrt{V_{S}^{2}+V_{N H}^{2}-2 V_{S} V_{N H} \cos \alpha}
$$

And $p d f$ of relative velocity can be derived as:

$$
\mathrm{f}_{\mathrm{V}}\left(\mathrm{R}_{\mathrm{V}}\right)=\frac{2 \mathrm{~V}}{\pi \sqrt{4 \mathrm{~V}^{2}-\mathrm{R}_{\mathrm{V}}^{2}}}
$$

Furthermore, it is required to have link duration between two nodes for the complete path duration estimation. $p d f$ for link duration is given below in :

$$
\mathrm{F}_{\mathrm{T}}\left(\mathrm{T}_{\mathrm{L}}\right)=\int_{0}^{\mathrm{V}}\left[\mathrm{D}_{\mathrm{L}}\right]\left[\frac{2 \mathrm{~V}}{\pi \sqrt{4 \mathrm{~V}^{2}-\mathrm{R}_{\mathrm{V}}^{2}}}\right] \mathrm{dR}_{\mathrm{V}}
$$

In THERA, GVs are responsible for calculating and maintaining the reliable links for every road segment. For the estimation of complete path duration between intersections, probability density function ( $p d f)$ is derived using Eq. 2, 4 and 5.

$$
T_{\text {LPath }}(\text { average })=\int_{0}^{\alpha} T_{L} f\left(T_{L}\right) d T_{L}
$$


Where $T_{L P a t h}$ represents the average path duration between the two ends of a road segment and $\mathrm{T}_{\mathrm{L}}$ is the link lifetime up to one hop node.

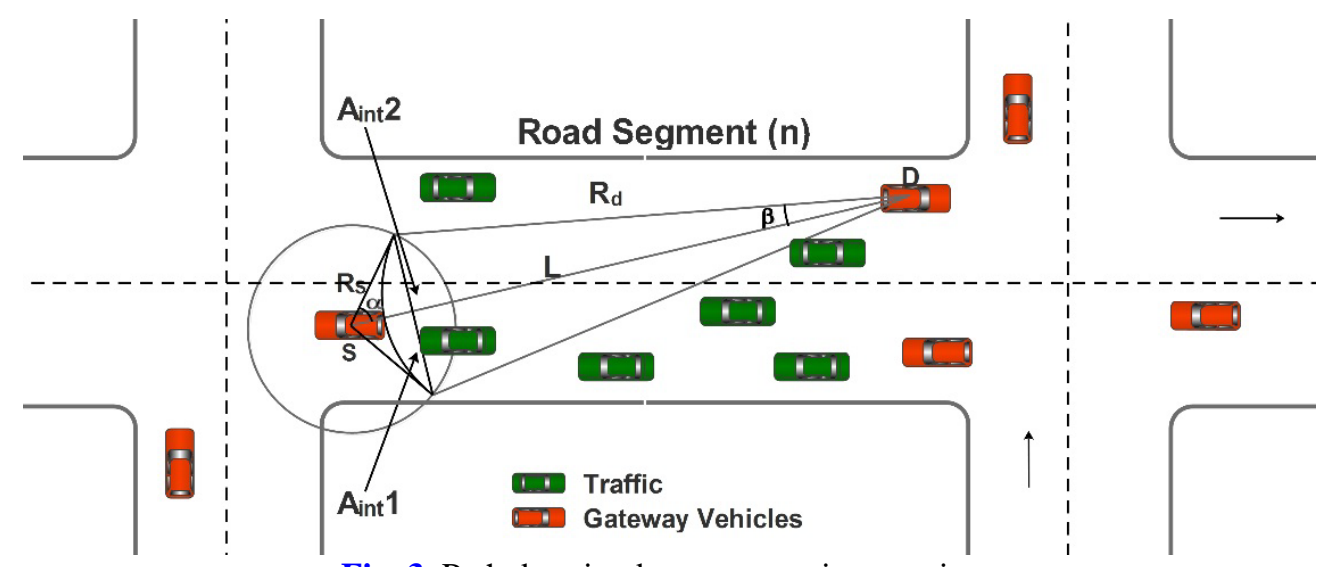

Fig. 3. Path duration between two intersections

Table 3. Variables and descriptions

\begin{tabular}{|l|l|}
\hline Variable & Description \\
\hline $\mathrm{S} / \mathrm{D}$ & Gateway vehicles (Source/destination) \\
\hline $\mathrm{L}$ & Total distance between two intersections/crossings \\
\hline $\mathrm{R}_{\mathrm{s}} / \mathrm{R}_{\mathrm{d}}$ & $\begin{array}{l}\text { Vehicle range (Source) / Distance between source's neighbor to } \\
\text { destination }\end{array}$ \\
\hline $\mathrm{A}_{\text {int }} 1 / \mathrm{A}_{\text {int }} 2$ & Area of intersection (1/2) \\
\hline $\mathrm{A}_{\text {Total }}$ & Expected area for next hop nod \\
\hline $\mathrm{D}_{\mathrm{L}}$ & Expected link distance between the source and a relay node \\
\hline $\mathrm{R}_{\mathrm{V}}$ & Relative velocity \\
\hline $\mathrm{V}_{\mathrm{S}}$ & Source node velocity \\
\hline $\mathrm{V}_{\mathrm{NH}}$ & Neighbor node velocity \\
\hline
\end{tabular}

\section{Experimental Classification Results and Analysis}

\subsection{Simulation Environment:}

In this study, network simulator 3 was used with the probabilistic Shadowing model. It is worth mentioning that, unlike the Two-ray Ground Reflection and the Free Space models, the probabilistic Shadowing model does not anticipate the gaining power as a deterministic behavior of space. Rather than that, it uses a numerical way to determine the total power and acknowledge multi-path dissemination impact. One of the main contribution of this paper is having a shortest and durable path for each road segment which is estimated using the cumulative distributed function using different parameters, which includes hop count, vehicle range, speed, and density. The reason for using probabilistic shadowing model is that it extends the ideal circle model to a richer statistic model and vehicles can probabilistically communicate with the neighbor vehicles only when near the edge of the communication range. However, in two-ray and free space model, the communication range is represented by an 
ideal circle. Before using the shadowing model, the user has to select the appropriate value for path loss $(\beta)$ and shadowing deviation according to their simulated environment. I have used $\beta=2.7$ (value of $\beta$ is 2 for free space propagation and is $2.7-5$ for an urban area) and shadowing deviation $=6.8$ for highway scenario and 5 for city scenario (can vary from 3 to 6 ).

In the Shadowing Model, the appropriate transmission range for the city scenario is set to 250 meters and 350 meters for the highway. For the evaluation purpose, two protocol rigorously evaluated that includes TCAR and CISRP. Using SUMO, the vehicle trace file was generated for an area of $1.3 \mathrm{Km}^{2}$. For the evaluation of city scenario, Gangnam road map is used, as shown in Fig. 10. However, the main highway with few intersections is considered for the evaluation of a highway scenario.

In order to validate our proposed protocol, we have compared it with TCAR [25] and CISRP [24] for a city and a highway scenario with three different vehicle densities:

- Low -25 vehicles/km or less

- Medium - 45 vehicles/km

- High - 60 vehicles/km or more

15 CBR sources are considered for simulation with a sending rate of $6 \mathrm{pkts} / \mathrm{s}$. 40 seconds before the simulation end, sources stop generating data packets. Every node remains inside the simulation area during the simulations (800 seconds). See Table 4, for more simulation details.

\subsection{Metrics}

For analyzing the performance of protocols assessed, we present the following metrics:

- Packet delivery ratio: PDR is the fraction of total packets generated by source vehicle that every protocol forwards it to the destination.

- Average data packet delay: Data packet delay is the difference of the time for which is stays in the network until it delivers successfully.

- Total Routing overhead: The number of communication data packets sent during the simulation.

- Link failure ratio (LFR): LFR is the proportion of data packet directed to the number of packets dropped.

- Path duration: Time for which a link remains active between source and destination for data transmission.

\subsection{Path duration}

In VANETs, choosing the shortest path for communication is not always feasible in case of path duration. Paths with more link residual life are preferred over the shorter link lifetime. Fig. 4 shows the path duration for highway scenario, as shown in Fig. 4(a), and city scenario, as shown in Fig. 4(b), with different densities of vehicles. TCAR protocol performs destitute in highway scenario as well as in the city scenario with the duration of 3-6 seconds and with vehicle density of 5-50. However, CISRP gives almost the same results when compared to TCAR with path duration of 3.2-6 seconds. This is because in both the protocols, the shortest path is maintained by the intermediate vehicles and a slight movement can causes link breakage. Note that THERA noticeably outperforms in terms of path duration and this is because of maintaining only durable paths inside the road segments by the gateway vehicles 
only. As we have discussed previously that paths with more duration are estimated and maintained proactively hence provides more stability and reliability.

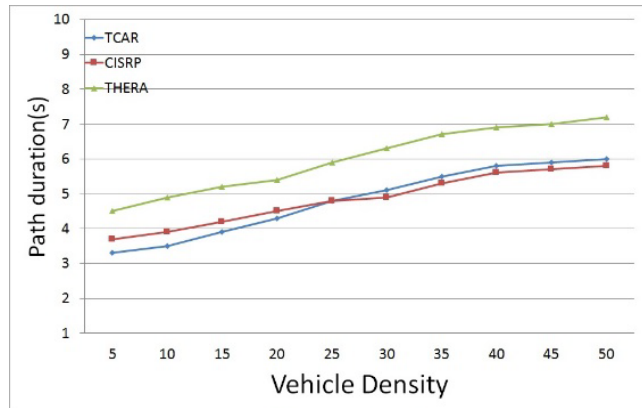

(a) City Scenario

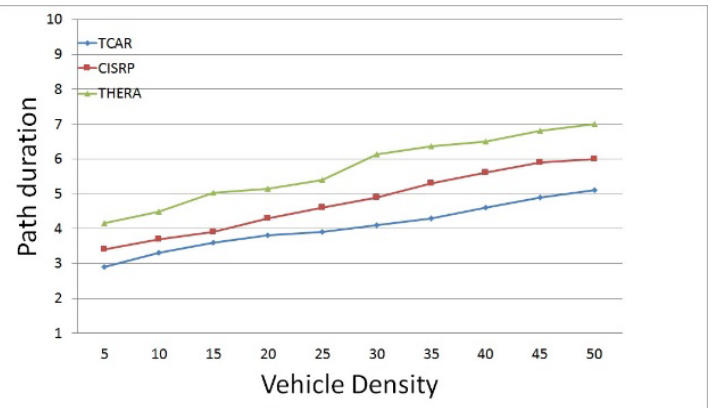

(b) Highway Scenario

Fig. 4. Path duration between two intersections

Table 4. Simulation Setup

\begin{tabular}{|c|c|}
\hline Parameters & Value \\
\hline \hline Simulation area & $2000 \times 2000 \mathrm{~m}$ \\
\hline Simulation Time & $800 \mathrm{~s}$ \\
\hline Total Number of Vehicles & More than 1000 \\
\hline Vehicles speed & $18 \mathrm{Km} / \mathrm{h}-120 \mathrm{Km} / \mathrm{h}$ \\
\hline Data packet size & 512 bytes \\
\hline Communication Range & 350 \\
\hline
\end{tabular}

\subsection{Link failure ratio}

Fig. 5(a) and Fig. 5(b) shows the ratio of communication link failure for a city as well as a highway scenario with increasing vehicle densities. It can be seen from the graph that THERA can have low failure ratio even in the presence of low vehicle densities and it can be related to maintaining shortest path by the gateway vehicles for each road segment. CISRP and TCAR perform poorly and has more failure ratio. The reason is that THERA selects neighbor vehicles with the same direction and almost zero relative velocity which enables durable communication links. Furthermore, THERA forwards the traffic on the stable and durable paths calculated by the GVs. This is only the reason that THERA shows higher performance than CISRP and TCAR.

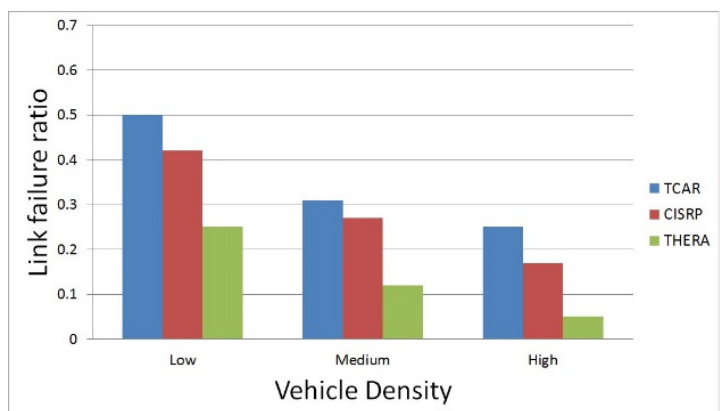

(a) City Scenario

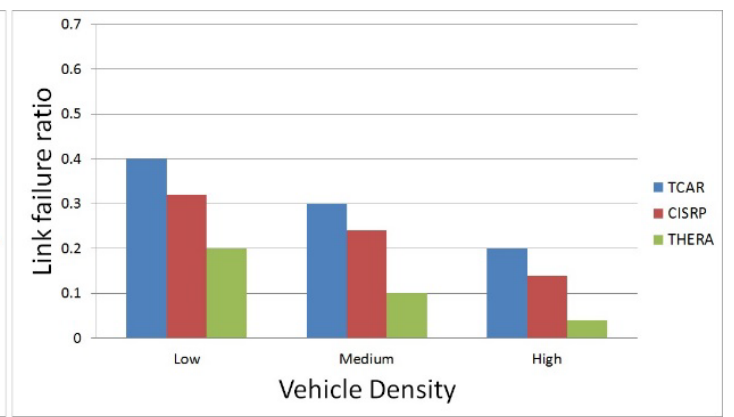

(b) Highway Scenario

Fig. 5. Link failure ratio 


\subsection{Routing Overhead}

Fig. 6(a) and Fig. 6(b) shows normalized routing overhead for TCAR, CISRP, and THERA. THERA generates less overhead at all simulated traffic densities. Further, unlike other protocols, THERA does not broadcast route discovery mechanism rather than it only forwards to GV. Routing overhead of TCAR mainly consists of finding a next forwarding section through macroscopic greedy selection strategy, however, in CISRP the routing overhead caused by the failed paths contributes significantly to the degraded performance. Whereas, in THERA, there is no broadcast for the destination discovery process. Discovery packets are sent only to the gateway vehicles. It can be seen from the results that THERA generates less routing overhead than other two protocols in both scenarios. It can be seen from the Fig. 6 that routing overhead increases for all the protocols as the vehicle density changes this is because the number of transmitted packets have a direct relation with the number of vehicles on roads.

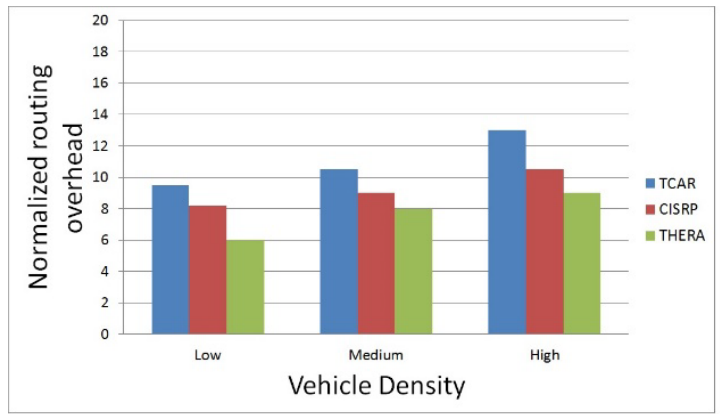

(a) City Scenario

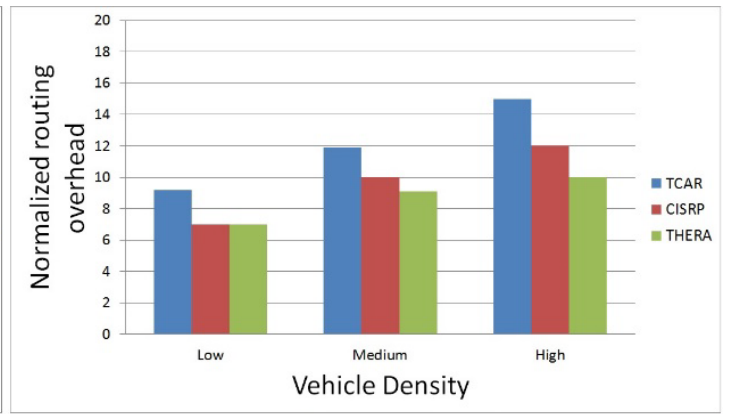

(b) Highway Scenario

Fig. 6. Routing Overhead

\subsection{End-to-End delay (Average)}

Fig. 7(a) and Fig. 7(b) demonstrates the end-to-end delay for TCAR, CISRP and THERA simulated for all vehicle densities. In both the scenarios (City, Highway), CISRP and TCAR behave impoverished. In THERA, a process of route discovery processed only once when data transmission is required. The route discovery process contributes some delay for the first time only. Once an entire path is known, it then further maintained by the GVs. Hence reduces the overall delay by adding some only for the first packet. The less hop count in the routing mechanism is required to reduce the end to end delay. This is done by the calculating the shortest path for each road segment by the GVs. Considering less number of hops by selecting a neighbor node at its maximum range is the reason that THERA performs better in both the scenarios which is not a case for other protocols. 


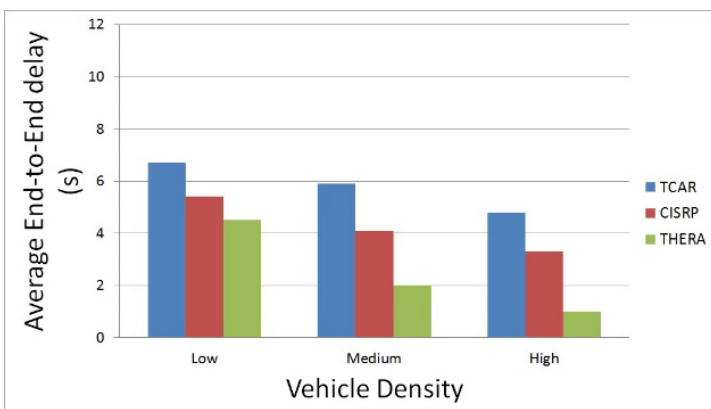

(a) City Scenario

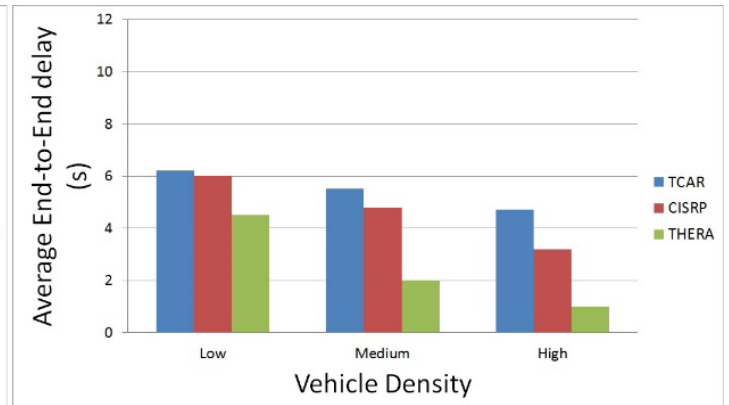

(b) Highway Scenario

Fig. 7. Average End-to-end delay

\subsection{Packet delivery ratio}

Packet delivery ratio with three vehicle densities are shown in Fig. 8(a) and Fig. 8(b) for both the scenarios, city, and highway, respectively. TCAR operates with bad performance in all the scenarios as it does not opt for a better route with the desired number of vehicles. It shows less than $70 \%$ delivery of data packets even in the denser network, as shown in Fig. 8. On the other hand, CISRP performs better than TCAR but it shows poor delivery ration than THERA because of the reason that it forwards the packet using the vehicle id, which is not the case for THERA, which is done using the road segment id instead. THERA demonstrates improved results than TCAR and CISRP despite the increased overhead to create and uphold the intra-road segment routing table.

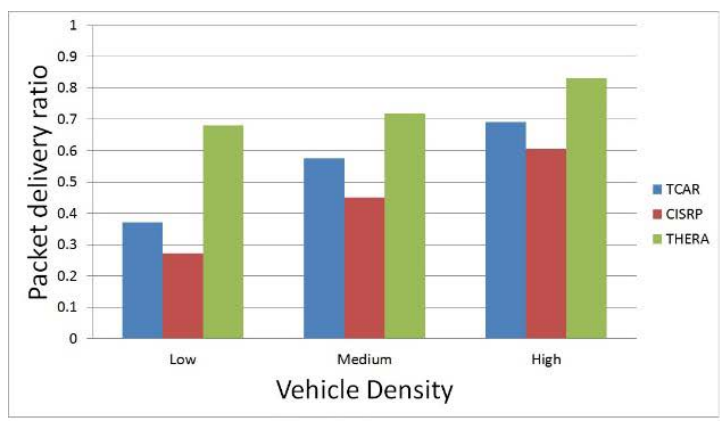

(a) City Scenario

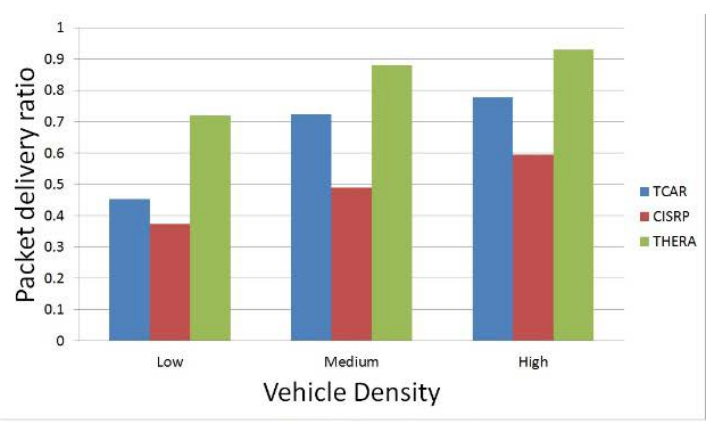

(b) Highway Scenario

Fig. 8. Packet Delivery ratio

\subsection{Routing overhead at Gateway vehicle:}

Normalized routing overhead for gateway vehicles are shown in Fig. 9(a) and Fig. 9(b) for both the scenarios, city, and highway, respectively. In THERA, GV performs significant task i.e. packet forwarding, RREQ, path estimation, maintaining shortest paths between intersections etc. In addition, GV also provides connectivity between two road segments. Considering all these aspects of GV in THERA, it shows a considerable difference in terms of routing overhead when compared to a normal vehicle in TCAR and CISRP. In addition, TCAR and CISRP start a new link discovery process whenever a connection fails between source and destination. However, in case of connection failure, THERA can deviate the data forwarding to alternative path due to the proactive routing between the intersections and by use of road segment ID rather than a vehicles ID. 


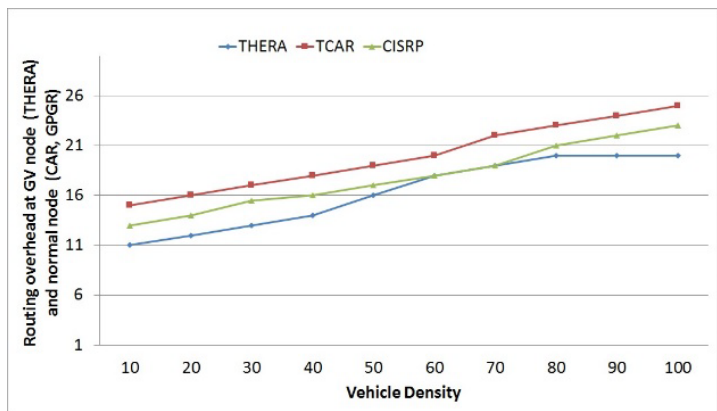

(a) City Scenario

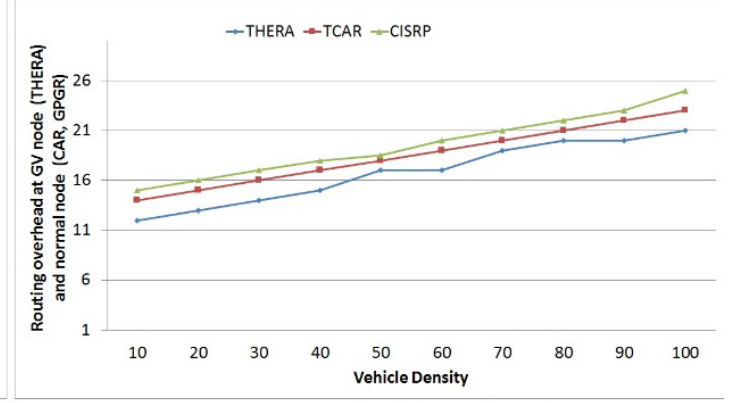

(b) Highway Scenario

Fig. 9. Routing overhead at Gateway nodes

\section{Conclusion}

In this study, we presented THERA, a new Road-Aware routing protocol for VANETs. THERA incorporate the characteristics of both the protocols, proactive and reactive for providing a reliable and low overhead routing approach for inter-vehicle communication. THERA uses an approach of two-level hierarchical road-aware routing to minimize the routing overhead rather than a network-wide broadcast destination discovery mechanism. THERA adapts to dynamic changes to enhance the packet delivery ratio by the use of road ID instead of vehicle ID in RREQ/RREP. The concept of gateway vehicle helps route discovery along with finding new routes on the fly in case of route failure.

For the evaluation, a realistic vehicular trace is generated with more than 1000 vehicles running on the roads of Gangnam, South Korea. The evaluation of THERA with TCAR and CISRP shows that THERA protocol bears a decent improvement in average data packet delay and packet delivery rate. A comparative analysis is performed for three protocols (TCAR, CISRP, and THERA) with different parameters. In the city scenario, CISRP shows more PDR than TCAR, whereas THERA shows 38\% more PDR than CISRP protocol. Furthermore, End-to-End delay for THERA is 2.46 seconds for the city and 2.60 seconds for highway, which is very small when compared to TCAR and CISRP.

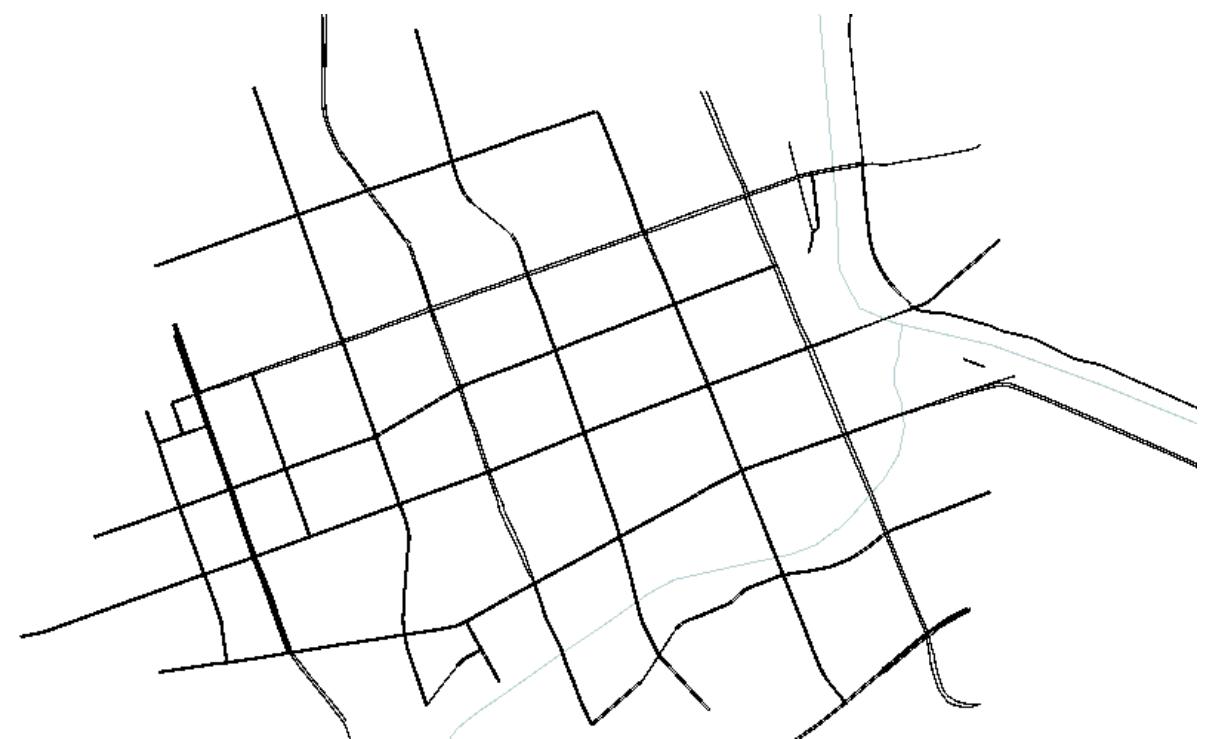

Fig. 10. Simulated Gangnam road map 


\section{Acknowledgement}

This research was supported by Basic Science Research Program through the National Research Foundation of Korea (NRF) funded by the Ministry of Education (NRF-2016R1D1A1B01016322).

This research was supported by the MSIT (Ministry of Science and ICT), Korea, under the ITRC (Information Technology Research Center) support program (IITP-2017-2017-0-01633) supervised by the IITP (Institute for Information \& communications Technology Promotion)

\section{References}

[1] H. Saleet et al., "Intersection-based geographical routing protocol for VANETs: A proposal and analysis,” IEEE Trans. Veh. Technol., vol. 60, no. 9, pp. 4560-4574, Nov. 2011. Article (CrossRef Link)

[2] Barba, Carolina Tripp, Luis Urquiza Aguiar, and Monica Aguilar Igartua, "Design and evaluation of GBSR-B, an improvement of GPSR for VANETs," IEEE Latin America Transactions, 11.4, 1083-1089, 2013. Article (CrossRef Link)

[3] Fonseca, António, and Teresa Vazão, "Applicability of position-based routing for VANET in highways and urban environment," Journal of Network and Computer Applications, 36.3, 961-973, 2013. Article (CrossRef Link)

[4] Abbasi, Irshad A., et al., "A traffic flow-oriented routing protocol for VANETs," EURASIP Journal on Wireless Communications and Networking, 2014.1, 121, 2014. Article (CrossRef Link)

[5] Zhu, Lina, et al., "Geographic routing in multilevel scenarios of vehicular ad hoc networks," IEEE Transactions on Vehicular Technology, 65.9, 7740-7753, 2016. Article (CrossRef Link)

[6] Li, Changle, Mengmeng Wang, and Lina Zhu, "Connectivity-sensed routing protocol for vehicular ad hoc networks: analysis and design,” International Journal of Distributed Sensor Networks, 11.8, 649037, 2016. Article (CrossRef Link)

[7] Salkuyeh, Mostafa Asgharpoor, and Bahman Abolhassani, "An adaptive multipath geographic routing for video transmission in urban VANETs," IEEE Transactions on Intelligent Transportation Systems, 17.10, 2822-2831, 2016. Article (CrossRef Link)

[8] Zhang, Hongli, and Qiang Zhang, "A Novel Road Topology-aware Routing in VANETs," Web Technologies and Applications. Springer International Publishing, 167-176, 2014. Article (CrossRef Link)

[9] Alsharif, Nizar, Sandra Céspedes, and Xuemin Shen, "iCAR: Intersection-based connectivity aware routing in vehicular ad hoc networks," in Proc. of Communications (ICC), 2013 IEEE International Conference on. IEEE, 2013. Article (CrossRef Link)

[10] Alsharif, Nizar, and Xuemin Sherman Shen, "iCARII: Intersection-based connectivity aware routing in vehicular networks," in Proc. of Communications (ICC), 2014 IEEE International Conference on. IEEE, 2014. Article (CrossRef Link)

[11] Tripp-Barba, Carolina, et al., "A multimetric, map-aware routing protocol for VANETs in urban areas,” Sensors, 14.2, 2199-2224, 2014. Article (CrossRef Link)

[12] Cai, Xuelian, et al., "LSGO: link state aware geographic opportunistic routing protocol for VANETs,” EURASIP Journal on Wireless Communications and Networking, 2014.1, 96, 2014. Article (CrossRef Link)

[13] Jabbarpour, Mohammad Reza, et al., "Performance analysis of V2V dynamic anchor position-based routing protocols,” Wireless Networks, 21.3, 911-929, 2015. Article (CrossRef Link)

[14] Akamatsu, Ryosuke, et al,"Adaptive delay-based geocast protocol for data dissemination in urban VANET," in Proc. of Mobile Computing and Ubiquitous Networking (ICMU), 2014 Seventh International Conference on. IEEE, 2014. Article (CrossRef Link) 
[15] Abbas, Muhammad Tahir, and Wang Cheol Song, "A path analysis of two-level hierarchical road. Aware routing in VANETs," in Proc. of Ubiquitous and Future Networks (ICUFN), 2017 Ninth International Conference on. IEEE, 2017. Article (CrossRef Link)

[16] M. H. Eiza, Q. Ni, T. Owens, and G. Min, "Investigation of routing reliability of vehicular ad hoc networks,” EURASIP J. Wireless Commun. Netw., vol. 2013, no. 1, p. 179, Jul. 2013. Article (CrossRef Link)

[17] Karagiannis, G.; Altintas, O.; Ekici, E.; Heijenk, G.; Jarupan, B.; Lin, K.; Weil, T., "Vehicular Networking: A Survey and Tutorial on Requirements, Architectures, Challenges, Standards and Solutions,” Communications Surveys and Tutorials, IEEE, vol.13, no.4, pp.584,616, 4th Quarter 2011. Article (CrossRef Link)

[18] Amjad, Zubair, Wang-Cheol Song, and Khi-Jung Ahn, "Two-level Hierarchical Routing based on Road Connectivity in VANETs," in Proc. of Industrial Engineering, Management Science and Application (ICIMSA), 2016 International Conference on. IEEE, 2016. Article (CrossRef Link)

[19] C. C. Consortium. www.car-2-car.org.

[20] Chen, Chen, et al., "A connectivity-aware intersection-based routing in VANETs," EURASIP Journal on Wireless Communications and Networking, 2014.1, 42, 2014. Article (CrossRef Link)

[21] Amjad, Zubair, Wang-Cheol Song, and Khi-Jung Ahn, "Context-Aware Routing for hovering information in Vehicular Ad-Hoc Networks," in Proc. of Network Operations and Management Symposium (APNOMS), 2016 18th Asia-Pacific. IEEE, 2016. Article (CrossRef Link)

[22] Chouhan, Tushar Singh, and Rajvardhan Somraj Deshmukh, "Analysis of DSDV, OLSR and AODV Routing Protocols in VANETS Scenario: Using NS3," in Proc. of Computational Intelligence and Communication Networks (CICN), 2015 International Conference on. IEEE, 2015. Article (CrossRef Link)

[23] Kamesh Namuduri, Ravi Pendse, "Analytical Estimation of Path Duration in Mobile Ad Hoc Networks,” IEEE SENSORS JOURNAL, vol. 12, no. 6, pp. 1828-1835, JUNE 2012. Article (CrossRef Link)

[24] Venkatramana, Dharani Kumari Nooji, Shylaja Banagiri Srikantaiah, and Jayalakshmi Moodabidri, "CISRP: connectivity-aware intersection-based shortest path routing protocol for VANETs in urban environments,” IET Networks, 7.3, 152-161, 2018. Article (CrossRef Link)

[25] Qin, Huabiao, and Cong Yu, "A road network connectivity aware routing protocol for Vehicular Ad Hoc Networks," in Proc. of Vehicular Electronics and Safety (ICVES), 2017 IEEE International Conference on. IEEE, 2017. Article (CrossRef Link)

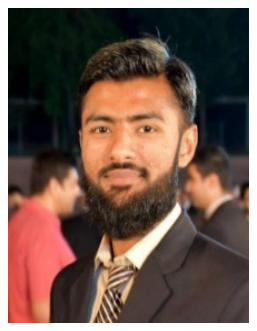

Muhammad Tahir received his B.E. degree in Computer engineering from E\&ME College, NUST, Pakistan, in 2015 and M.S degree in Computer Engineering from Jeju National University, South Korea. He worked as a Research Assistant at Center for Advance Research in Engineering (CARE) from 2014-2015. His current research interests include Future Networks, 5G, VANETs, SDN \& NFV, and Information Security.

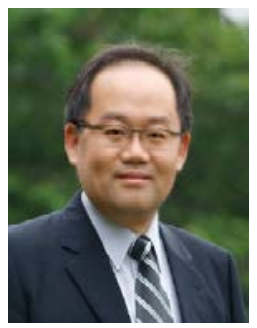

Wang-Cheol Song received B.S. degree in Food Engineering and Electronics from Yonsei University, Seoul, Korea in 1986 and 1989, respectively. And M.S. and Ph.D. in Electronics studies from Yonsei University, Seoul, Korea, in 1991 and 1995, respectively. Since 1996 he has been working at Jeju National University. His research interests include VANETs and MANETs, Software Defined Networks, network security, and network management 